\title{
Einleitung: Gemeinwohl und Gemeinsinn als politisch-soziale Leitbegriffe
}

Die in der Antike geprägten Begriffe Gemeinwohl und Gemeinsinn waren semantisch nie eindeutig festgelegt, und politisch blieben sie immer umstritten, aber gerade wegen ihrer wechselnden Bedeutung, dem Wandel ihrer orientierenden Funktion, schließlich auch wegen der verschiedenen politischen Optionen, die sich mit ihnen verbanden, konnten sie in traditionalen Gesellschaften und während des Übergangs zur Moderne zu politisch-sozialen Leitbegriffen avancieren. Ob und inwieweit sie auch in modernen Gesellschaften diese Rolle spielen können, ist umstritten, wird doch der Gemeinwohlbegriff von vielen substanzialistisch verstanden, und ein solcher Begriff, so der Tenor, sei in modernen pluralistischen Gesellschaften, in denen komplexe Verfahren und Institutionen dominieren, kaum angemessen verwendbar. Zudem seien die Akteure moderner Gesellschaft als rationale Nutzenmaximierer zu begreifen, die für ein Allgemeinwohl jenseits ihrer Eigeninteressen kaum Verständnis hätten, falls es sich nicht als $\mathrm{Ne}$ benfolge ihrer Präferenzentscheidungen ergäbe. Die Konsequenz lautet: Als Teil alteuropäischer Semantik mögen Gemeinwohl und Gemeinsinn in traditionalen Gesellschaften bedeutsam gewesen sein; in modernen Gesellschaften haben sie weder Funktion noch Bedeutung. Aber ist dies tatsächlich eine zutreffende Beobachtung?

Den Ausgangspunkt für die Thematisierung von Gemeinwohl und Gemeinsinn durch eine gleichnamige Arbeitsgruppe der Berlin-Brandenburgischen Akademie der Wissenschaften bildeten gegenteilige Beobachtungen; daß nämlich die Begrifflichkeit des Gemeinwohls entgegen der referierten Erwartung seit zwei Jahrzehnten einen neuerlichen Aufschwung genommen hat und in den jüngsten sozialphilosophischen wie politiktheoretischen Debatten eine nicht unerhebliche Rolle spielt. Es sind insbesondere zwei Problemkreise, in deren Zuge sie Karriere machte: der Umbau des Sozialstaates und die Voraussetzungen der Demokratie. ' Der Staat, der, zumal in Deutschland, lange Zeit als Hüter, Interpret und Durchsetzungsinstanz des Gemeinwohls galt, hat dieses Monopol weitgehend verloren oder von sich aus abgegeben. Im gegenwärtigen Staat - gleich, ob man ihn als kooperativen Staat, als schlanken Staat, der sich auf seine Kernaufgaben zurückzieht oder als aktivierenden Staat faßt - sind andere Akteure im Spiel als die

\footnotetext{
1 Vgl. exemplarisch Alemann u.a. 1999.
} 
herkömmlich mit der Gemeinwohlwahrung betrauten Beamten, und diese Akteure reklamieren nun die Verfügung über das Gemeinwohl für sich. Ebenso häufig findet sich das Gemeinwohl als politische Vokabel, wenn die Möglichkeiten des Erhalts oder Ausbaus der Massendemokratie im Medienzeitalter diskutiert werden. Die Themen reichen hier vom Bürgerengagement über Dezentralisierung, Föderalisierung und Kommunalisierung bis hin zu politisch-moralischen Forderungen an die politische Elite. ${ }^{2}$ All dies spricht für die universelle Verwendbarkeit der Begriffe, aber auch eine inhaltliche Vielfalt von Interessen, die mit ihnen verbunden werden können. Von dieser Überlegung aus erschien es uns sinnvoll, die historischen Traditionsbestände der Gemeinwohlsemantik neu auszuleuchten und auf ihre Gehalte hin zu befragen.

Das Ziel des vorliegenden historischen Bandes bildet die Rekonstruktion der beiden Leitbegriffe und ihrer Transformationen. Die Auseinandersetzung mit der Vielfalt dieser Semantik führt zum Verständnis der fortdauernden Bedeutung dieser Begriffe in den jüngeren Debatten. Der Band endet zeitlich mit dem langen 19. Jahrhundert, dessen Privilegierung einzelner akademischer Disziplinen bei der Auslegung des Gemeinwohls heute fremdartig anmutet. Nach den Totalitarismen des 20. Jahrhunderts, die letztlich im Namen von Gemeinwohl- und Gemeinschaftsideen erfolgten, ist jede substanzialistische Bestimmung dieser Begriffe jedoch desavouiert. Diese Ideen sind nun weltweit einer Fundamentalpluralisierung und -prozeduralisierung ausgesetzt, in der sie weiter an Bestimmtheit verlieren werden, aber Bestandteil der politischen Sprache demokratischer Regime bleiben. Von dieser Diagnose ausgehend, können Gemeinwohl und Gemeinsinn als politische Leitbegriffe mit großer Attraktivität für politische Akteure analysiert werden. Die folgenden Überlegungen skizzieren methodische und inhaltliche Gedanken sowie das begriffliche Instrumentarium für solche Analysen und führen in grundlegende Fragen des semantischen Wandels von Gemeinwohl und Gemeinsinn ein.

\section{Wiederaufnahme der Begriffe Gemeinwohl und Gemeinsinn}

Akademisch hat sich die Revitalisierung von Gemeinwohl und Gemeinsinn auf drei Feldern vollzogen. Zunächst ist die mit den beiden Begriffen bezeichnete Problematik in der Debatte zwischen Liberalen und Kommunitaristen zum Thema geworden, die anfangs der 1980er Jahre einsetzte. In dieser, zunächst vornehmlich amerikanischen Selbstverständigungsdebatte über sozio-moralische Aspekte der Demokratie, ist von den Kommunitaristen nach den subjektiven Voraussetzungen demokratischer Akteure gefragt worden, d.h. nach deren Sozialisation, demokratischen Kompetenzen, Werten und Tugenden. Dabei ist auf einen starken Begriff des Bürgers rekurriert worden, der nicht nur passiv als Konsument und purer Wähler gefaßt wird. ${ }^{3}$ Darüber hinaus wurden Ziele der Demokratie anhand von Konzepten der guten Ordnung und des guten Lebens debattiert. ${ }^{4}$ Diese Debatte, die Mitte der 1980er Jahre Europa erreichte und nach dem

2 Dazu Münkler 2000 sowie Münkler/Krause 2001.

3 Zur Debatte vgl. Honneth 1993 und Bell 1993; zum Bürgerbegriff vgl. Gebhardt/Münkler 1993, sowie Münkler 1997.

4 Vgl. Walzer 1992, v.a. Kap. 2. 
Fall der Mauer noch einmal Auftrieb erhielt, hat implizit einen neuen Zugang zur Problematik des Gemeinwohls eröffnet, da sie das Gemeinwohl eng mit der Frage des Gemeinsinns der Bürger verband. Ein aktueller Ausläufer ist die Diskussion um Robert Putnams Studie Bowling Alone. ${ }^{5}$ Putnam hat für die USA einen Verfall des Sozialkapitals diagnostiziert, der den Unterbau der Demokratie, die Vielfalt der zivilen Assoziationen betrifft. Seine Diagnose fand weltweit großes Echo und führte gelegentlich zur Aktualisierung eines einfachen Gegensatzes von Gemeinwohl und Privatwohl. Dagegen stellt Putnam in seiner Deutung des Sozialvermögens gerade auf die Verbindung von privatem und öffentlichem Nutzen ab: „Social Capital can thus be simultaneously a ,private good" and a ,public good “ “. 6 Soziales Kapital wird hier als privates Vermögen gefaßt, dessen Nutzung im öffentlichen Interesse liegt.

Zum anderen ist für die jüngere Verbindung von Gemeinwohl und Gemeinsinn eine in den Sozialwissenschaften forcierte, formelle und institutionelle Dimensionen einbeziehende Fassung der Pluralisierung und Prozeduralisierung von Gemeinwohl wesentlich. Ein Indikator dafür ist, daß ein namhafter Politikwissenschaftler wie Robert Dahl selbstverständlich davon ausgeht, daß das „common good“ nur als Prozeß gefaßt werden kann. Dahl scheut auch nicht vor einer Definition zurück:

"The common good among the members of a group is what the members would choose if they
possessed the fullest attainable understanding of the experience that would result from their
choice and its most relevant alternatives. Because enlightened understanding is required, I
would propose to incorporate to acquire enlightened understanding as essential also to the
meaning of the common good. Still further, the rights and opportunities of the democratic proc-
ess are elements of the common good. Even more broadly, because the institutions of polyarchy
are necessary in order to employ the democratic process on a large scale, in a unit as large as a
country all institutions of polyarchy should also be counted as elements of the common good."

Was Dahls Definition kennzeichnet, ist die Benennung systemischer Voraussetzungen des Gemeinwohls. Fritz Scharpf hat diesen Gedanken mit Bezug auf die Legitimitätsproblematik wie folgt formuliert:

„Die input-orientierte Perspektive betont die ,Herrschaft durch das Volk. Politische Entscheidungen sind legitim, wenn und weil sie den ,Willen des Volkes* widerspiegeln - das heißt, wenn sie von den authentischen Präferenzen der Mitglieder einer Gemeinschaft abgeleitet werden können. Im Unterschied dazu stellt die output-orientierte Perspektive den Aspekt der ,Herrschaft für das Volk' in den Vordergrund. Danach sind politische Entscheidungen legitim, wenn und weil sie auf wirksame Weise das allgemeine Wohl im jeweiligen Gemeinwesen fördern." ${ }^{* 8}$

Zurecht sind für Dahl und Scharpf Gemeinwohl und Gemeinsinn nicht nur als einfache Handlungsorientierungen wichtig, sondern sie zielen zugleich auf die Reproduktion

\footnotetext{
5 Vgl. Putnam 1995, 2000; zum Echo vgl. Skocpol 1997, Ladd 1999, Deth et al. 1999, Offe 1999, Klages 1999, Putnam 2001.

${ }^{6}$ Putnam 2000, S. 20.

7 Dahl 1989, S. 307f.

${ }^{8}$ Scharpf 1999, S. 16; vgl. auch S. 168.
} 
zentraler subjektiver Voraussetzungen der Demokratie und demokratischer Institutionen.

Fand die Debatte der Liberalen und Kommunitaristen in Philosophie, Soziologie, Politikwissenschaft und Historiographie ein lebhaftes Echo, so kam es schließlich auch auf dem Feld der Ökonomie zu einer Umorientierung, die Albert Hirschman pointiert hat: „Die Wiederbelebung und Rehabilitierung des Gemeinsinnes setzte zunächst in der Mikroökonomie ein". ${ }^{9}$ Schon in den späten 50er und 60er Jahren sei anhand von Kooperations- und Wettbewerbsproblemen die Frage nach den subjektiven Voraussetzungen von Kooperation aufgekommen. Damit sind unter anderem ethische Regulierungen gemeint, mit denen Berufsgruppen die Ausbeutung von Informationsvorsprüngen im Rahmen von Selbstbeschränkungen verhindern können. Substanziell ist davon die Problematik des Vertrauens betroffen, die bei jeder Art von Vertragsbeziehungen eine Rolle spielt und nicht vollständig in rationale Interessenkalkulationen aufgelöst werden kann. Die eigentliche Rehabilitierung von Moral und Gemeinsinn hat sich laut Hirschman jedoch in der Makroökonomie vollzogen, und zwar auf den Gebieten der Wirtschaftspolitik, der kooperativen Beziehungen zwischen staatlichen Institutionen, gesellschaftlichen Akteuren und Verbänden sowie im Bereich regionaler Ökonomien. Insgesamt hält Hirschman nur ein komplexes Modell menschlichen Verhaltens, das die moralischen Seiten, Leidenschaften - Emotionen und Eigennutz inklusive - berücksichtigt, für eine geeignete Grundlage der Modellierung von Wirtschaftsprozessen. Es verwundert nicht, daß er seine Kritik an Mancur Olsons Handlungskonzept, das den homo oeconomicus universalisiert, wie folgt zusammenfaßt: Statt der Logik kollektiven Handelns habe Olson nur dessen Unlogik untersucht. Wie erkläre sich, daß trotz einfacher Nutzenkalküle und Trittbrettfahrerei auch mannigfach kollektives Handeln realisiert werde? Gerade dafür seien, so Hirschman, Moral und sozio-moralische Ressourcen für wirtschaftliches Handeln relevant, wobei er für eine eher skeptische Berücksichtigung dieser Faktoren plädiert. ${ }^{10}$

Mit Blick auf die angedeuteten Debatten erscheint es angezeigt, Gemeinwohl und Gemeinsinn als komplementäre Kategorien zu thematisieren. Dabei sind verschiedene Gesichtspunkte relevant. Zunächst gilt es, deutlich zu unterscheiden zwischen den materialen Gehalten von Gemeinwohlkonzepten und substanzialistischen Konzepten, die ein objektives, gar transhistorisches Gemeinwohl unterstellen. Materiale Gehalte können substanzialistisch, aber auch Ausdruck eines zeitweiligen Konsenses der Akteure sein. Erst jenseits eines substanzialistischen Verständnisses rücken praktische und rhetorische Definitionsprozesse von Gemeinwohl und deren Bezug auf den Gemeinsinn von Akteuren als Untersuchungsgegenstand in den Vordergrund. Wo dies geschieht, geht es zumeist um die Frage, mit welchen Gehalten die „Leerformel" Gemeinwohl gefüllt wird. $\mathrm{Zu}$ beachten sind darüber hinaus eine Reihe an Substitutbegriffen für Gemeinwohl, wie öffentliches Interesse, Allgemeininteresse, beziehungsweise für Gemeinsinn, wie be-

\footnotetext{
Hirschman 1989, S. 95.

${ }^{10}$ Hirschman 1989, S. 98ff., zur Kritik an Olson vgl. Hirschman 1997, S. 74f.; daß Gemeinwohlanforderungen wenig enttäuschungsresistent sind und es infolgedessen zu einem zyklischen Auf und Ab des Engagements der Bürger kommen kann, hat er in Hirschman 1984, S. 9ff. gezeigt.
} 
stimmte Tugenden, Solidarität, Verantwortungsbewußtsein u.a.m. ${ }^{11}$ In diesem weiten Kontext lassen sich Gemeinwohl und Gemeinsinn in einer ersten allgemeinen Bestimmung wie folgt verstehen:

Gemeinsinn kann als eine motivationale Handlungsdisposition von Bürgern und politisch-gesellschaftlichen Akteuren begriffen werden, die eine prinzipiell knappe soziomoralische Ressource darstellt. Sie bildet die „subjektive“ Seite gemeinwohlorientierten Handelns, die nur partiell im Handeln selbst reproduziert werden kann. Gemeinwohl ist das normative Ideal, das uns implizit auch sagt, wieviel Gemeinsinn wir aufbringen müssen, um die im Gemeinwohlbegriff umschriebenen Resultate des politischen Prozesses zu erreichen. Es bedarf aber eines Mindestmaßes an Gemeinsinn, damit wir überhaupt motiviert sind, uns für ein normatives Gemeinwohl-Ideal zu interessieren. ${ }^{12}$ Gemeinwohl zielt als Begriff auf gemeinsame Ziele, Kooperationseffekte und ein Handeln, das auf den Erhalt der jeweiligen Gemeinschaft und die Reproduktion ihrer Voraussetzungen gerichtet ist. So verstanden, rücken verschiedene spannungsvolle Relationen zwischen Gemeinwohl und Gemeinsinn in den Blick, von denen hier nur eine aktuelle erwähnt werden soll: Bei der Aktivierung von Gemeinwohlpostulaten ist die Größe der Bezugsgruppe (Gemeinde, Region, Nationalstaat, EU) zu bedenken, da sowohl die Identifikation als auch die Erkennbarkeit und Bewertbarkeit dessen, was als Gemeinwohl gelten soll, mit wachsender Größe der Bezugsgruppe bzw. Struktur prekärer wird. ${ }^{13}$ Gerade an den Vorstellungen von Gemeinwohl und Gemeinsinn zeigt sich der moralphilosophisch wenig befriedigende Befund, daß mit dem Grad der Universalisierung von Anforderungen deren Verbindlichkeit und Verpflichtungscharakter schwindet - und umgekehrt.

\section{Dimensionen von Gemeinwohl und Gemeinsinn}

Die verbreitete Kontrastierung von alteuropäischer und moderner Semantik verstellt oft in zweierlei Richtung den Blick: Zum einen kann sie die erstaunliche Überlebenskraft alteuropäischer Begriffe kaum erklären, denn die Abweisung dieser Begriffe in den Bereich von Reflexionstheorien vermag weder deren fortbestehende theoretischen $\mathrm{Ge}$ halte noch ihre politischen Funktionen, etwa in der jüngsten Debatte zwischen Liberalen und Kommunitaristen, verständlich zu machen. Man muß hier schon mehr als eine Tradierung alter Begriffe und Kontinuierung alteuropäischer Semantik erkennen können, wenn man die sachlichen wie rhetorisch-politischen Aspekte dieser Debatte erschließen will. Die typologische Gegenüberstellung von alteuropäischer und moderner Semantik versperrt auch den Blick für die Vielfalt an Variationen dieser Semantik und ihrer mannigfachen Transformationen. Trotz einer Reihe verdienstvoller Einzelstudien ${ }^{14}$ ist bisher im einzelnen nicht bekannt, wie sich diese Semantik entwickelt hat.

11 Vgl. Münkler 1996, passim.

12 Vgl. Fischer 2000, S. 139 f.

13 Münkler/Fischer 1999, S. 238f.

14 Vgl. Hibst 1991, Kempshall 1999, Gunn 1969, Miller 1994, Koslowski 1999, Maier 1966. 
Man kann drei Dimensionen der Thematisierung von Gemeinwohl und Gemeinsinn unterscheiden: zunächst als politische Ideen, denen eine retrospektiv-explikative wie eine perspektivische, also handlungsleitende Funktion zukommt. Gemeinwohl und Gemeinsinn fungieren weiterhin als rhetorisch-politische Kategorien, die im Rahmen historischer Semantiken verwandt werden, und schließlich spielen sie in Verbindung mit Institutionen und Verfahren, und zwar vor allem politisch-juridischer Art, eine bedeutende Rolle. Wenn man diese Dimensionen unterscheidet und den Wandel der Semantiken von Gemeinwohl und Gemeinsinn im skizzierten Zusammenhang thematisiert, können auch Antworten auf die Frage nach ihrer erstaunlichen Überlebensfähigkeit gegeben werden. Dabei verdient die Ebene der Gemeinwohlrhetorik eine besondere Beachtung, denn hier kommt die praktisch-öffentliche Dimension ins Spiel, die sowohl mit den politischen Ideen und ihrer Wirksamkeit verbunden ist als auch einen Zugang $\mathrm{zu}$ institutionellen Verfahren und Praktiken eröffnet. An den rhetorischen Verwendungsweisen von „Gemeinwohl“ lassen sich allgemein folgende Varianten fixieren: Die Gemeinwohlrhetorik kann inklusiv bzw. inkludierend oder exklusiv bzw. exkludierend verwendet werden, und zwar jeweils mit oder ohne Appell an den Gemeinsinn. In beiden Fällen werden Ansprüche erhoben und abgewehrt. Zudem kann man bei positiven wie negativen Nutzungen deutlich zwischen Protagonisten und Adressaten der Ansprüche unterscheiden (von „oben“, vom Staat, der Regierung; von „unten“, von Bewegungen, Bürgern usw.). Wenn das Gemeinwohl von „oben“" interpretiert wird, ist sowohl eine Passivierung oder gezielte Aktivierung der Bürger denkbar, die sowohl auf direkte als auch indirekte Weise intendiert werden kann. D.h. mit der Rhetorik sind spezifische Niveaus der Anforderungen an Bürger und Akteure verbunden, und dabei geht es um das Ausmaß der politisch-moralischen Zumutungen. Allgemein gefaßt kann man zwischen einer Überforderung und einer Unterforderung der Bürger unterscheiden, die in einer liberalen Demokratie gleichermaßen zum Absinken des Engagements und zur Erosion des Gemeinsinns führen können.

Wenn man vom Gemeinwohl als rhetorisch-politischer Kategorie spricht, muß man nicht nur dessen Komplementärbegriffe, sondern auch konkurrierende Abgrenzungsbegriffe in die Überlegung einbeziehen. Klassisch ist der Gegensatz von Gemeinwohl und Eigennutz, der in der politischen Ideengeschichte, nicht zuletzt unter dem Einfluß der politischen Theorie des Aristoteles, lange Zeit eine zentrale Rolle spielte, bis er durch den Liberalismus, der das Gemeinwohl als Resultante von eigennützigem Handeln faßte, in den Hintergrund gedrängt wurde. Zurecht ist damit die einfache Opposition beider Begriffe aufgebrochen worden, jedoch ist kaum zu übersehen, daß es nach wie vor Sinn macht, am Gegensatz von Gemein- und Eigennutz festzuhalten. Allerdings bilden die häufig anzutreffenden Vermittlungs- und Mischformen von individuellen, kollektiven und allgemeinen gesellschaftlichen Interessen den wirklich schwierigen Forschungsgegenstand. Dieser Mischformen wird man nur habhaft, wenn man Gegenbegriffe zum Gemeinwohl berücksichtigt. Zum einen können Rechte als Abgrenzungsbegriff zum Gemeinwohl gefaßt werden; enthalten erstere fixierte Normen, so hebt das Gemeinwohl auf ein Sollen ab, das zusätzliche Bindungen betont. Zum anderen ist das Gemeinwohl in negativen Bestimmungen oft besser zu fassen als in positiven Definitionen, nämlich in Varianten gemeinwohlschädigenden Verhaltens, wie dem Egoismus von Individuen, 
Gruppen und Koalitionen, Korruption u.a.m. ${ }^{15}$ Solche Negativdiagnosen werden von zumindest impliziten Gemeinwohlvorstellungen getragen und nehmen nicht selten explizit auf das Erfordernis von Gemeinsinn Bezug. Je nachdem, welche Gegenbegriffe man einsetzt, fallen nicht nur die Rhetoriken der Mobilisierung gemeinwohlorientierten Handelns verschieden aus, sondern es rücken auch andere Konsequenzen des Handelns in den Vordergrund.

Im Kontext dieser generellen Überlegungen sollen nun einige begriffliche und ideengeschichtliche Perspektiven der Transformation der Semantik von Gemeinwohl und Gemeinsinn im Zusammenhang umrissen werden. Gerade dadurch läßt sich verdeutlichen, daß diese Begriffe vielfältig operational eingesetzt und in der Moderne innerhalb unterschiedlicher theoretischer Strömungen und politischer Verwendungsweisen wirksam wurden. Drei Gesichtspunkte seien hier kurz erwähnt. Zum einen soll gezeigt werden, daß in der Antike und der auf sie zurückgreifenden Renaissance die klassische Begrifflichkeit und ihre Wiederaufnahme komplexer angelegt waren, als in der Regel angenommen wird. Zum anderen ist zu skizzieren, daß der Übergang von alteuropäischer zu moderner Semantik nicht nur langwierig war, sondern sich im Geflecht verschiedener Strömungen vollzog. Schließlich geht es darum, unterhalb des Paradigmenwechsels von einem substanzialistischen zu einem nicht-substanzialistischen Verständnis des Gemeinwohls eine Reihe von Transformationen der Gemeinwohlsemantik in der Moderne aufzuzeigen.

\section{Historische Transformationen der Gemeinwohlsemantik}

Bevor auf die Ideengeschichte und die historischen Transformationen der Semantik eingegangen werden kann, ist zunächst eine Bemerkung zum Verhältnis von Gemeinwohl und Gerechtigkeit nötig. Die Dominanz von Gerechtigkeit als politischem Leitbegriff ist ein jüngeres Phänomen, das auf der Prägung des Begriffs „sozialer Gerechtigkeit" in der 2. Hälfte des 19. Jahrhunderts ruht, aber erst in den sich ausweitenden akademischen und politischen Diskursen im letzten Drittel des 20. Jahrhunderts in Erscheinung trat. ${ }^{16}$ In den Konzepten der guten Ordnungen, wie sie das politische Denken lange beherrschten, waren Gerechtigkeit und Gemeinwohl dagegen untrennbar miteinander verwoben. Eine generelle Differenz der beiden Begriffe läßt sich nur hinsichtlich ihrer Extension ausmachen: Während Gerechtigkeit als universalistische Norm gefaßt werden kann, ist Gemeinwohl immer auf eine begrenzte Gemeinschaft bezogen und von dieser nicht abtrennbar. Insofern ist Gemeinwohl eher ein partikularistisches Konzept, das sich allerdings durchaus auf eine gegliederte Ordnung von Gemeinschaften erstrecken kann. ${ }^{17}$ Es ist der konkrete Bezug auf Referenzgemeinschaften, der auch den bereits entwickelten systematischen Zusammenhang mit dem Gemeinsinn konkreter Akteure eröffnet.

\footnotetext{
15. Vgl. dazu Münkler/Fischer/Bluhm 2000, S. 425-440.

$16 \mathrm{Vgl}$. Hofmann 2000, S. 193-215.

17 Vgl. in ähnlichem Sinne Kaufmann 1999, S. 102-123.
} 
Ein grundsätzlicher Wandel in der Vorstellung von Gemeinwohl erfolgt in der Moderne, indem der seit den frühen antiken Begriffsprägungen dominierende Gegensatz von Privat- und Gemeinwohl dahingehend umgedeutet wird, daß das Gemeinwohl als Resultante des Verfolgens von privatem Wohl begriffen wird. Die Voraussetzungen dieses Wandels sind vielfältig. Zum einen spielt die in der Renaissance anhebende Säkularisierung der Politikauffassung eine Rolle, denn die Ablösung von der Theologie ermöglicht einen neuen Zugang zu Gemeinwohl und Gemeinsinn. Zum anderen kommt, damit einhergehend, eine neue Sichtweise von Politik zum Tragen, die, bezogen auf den institutionellen Flächenstaat, Politik in Zweck- und Funktionsbestimmungen zu fassen versucht. Die Konzepte von Staatsräson, Souveränität und Wohlfahrt bildeten den Rahmen, in dem Gemeinwohl und Gemeinnützigkeit neu und oft jenseits substanzialistischer philosophisch-theologischer Theoreme bestimmt werden konnte. Säkularisierung und Entsubstanzialisierung sind allerdings nur ein sehr grobes Schema; hinzu kommt, daß die Zweck- und Funktionsbestimmungen der gerechten, gemeinnützigen Ordnung, die geforderten Handlungskompetenzen der politischen Akteure und die Art der institutionellen Ordnung in veränderten Kontexten und Konstellationen des politischen Denkens stets neu arrangiert wurden. Mit Blick auf den Wandel der Gemeinwohlsemantik in praktisch-institutionellen Kontexten kann man sich kreuzende und abwechselnde Prozesse der Kommunalisierung, Verstaatlichung, Entstaatlichung, Nationalisierung und Entnationalisierung des Gemeinwohls unterscheiden. All dies sind zugleich Formen der politischen Grenzziehung bzw. Grenzverschiebung, bei denen neben der Referenz der Loyalität vor allem auch die Reichweite der Solidarität bzw. der Solidaritätszumutungen verändert wird. Aber bei diesem Wandel spielen nicht nur veränderte Bezugsgemeinschaften eine Rolle, sondern auch Neujustierungen des Verhältnisses von Privatem und Öffentlichem, der Wandel in Verfahren der Definition des Gemeinwohls und Veränderungen der Formen der Artikulation des Gemeinsinns.

\section{Antike Varianten}

Schon in der Antike war die Bestimmung des Gemeinwohlbegriffs weniger eindeutig als oft angenommen. Dies wird deutlich, wenn man die Untersuchung nicht auf die Klassiker der politischen Philosophie beschränkt, sondern auch die Verwendung von Gemeinwohl- und Gemeinsinnformeln in politischen Reden berücksichtigt. Zum Wortfeld von Gemeinwohl gehören, wie Guido Kirner zeigt, das Wohl der Stadt, die gute stabile Ordnung u.a.m. Insbesondere anhand des Thukydides läßt sich der vielfältige operationale Gebrauch des Gemeinwohlbegriffs aufzeigen: Er legt ihn verschiedenen Rednern in den Mund, wahrt zugleich aus machtanalytischer Perspektive aber Distanz zu den im Namen des Gemeinwohls, nach innen wie außen geltend gemachten Ansinnen, so daß sich bei ihm schon erste Ansätze eines ideologiekritischen Blicks auf Gemeinwohlrhetoriken ausmachen lassen.

Die Leistung von Platon und Aristoteles erscheint vor diesem Hintergrund als Systematisierung, Vereindeutigung, aber damit auch Verengung einer variablen politischen Begrifflichkeit. Dabei nimmt insbesondere Platon eine Substanzialisierung des Gemeinwohlbegriffs zu einem objektiven Gut vor, während Aristoteles in Verbindung mit seinem Ideal der rechten Mitte bzw. der gemischten Verfassung, eine Verknüpfung von 
Gemeinwohl und Gemeinsinn vornimmt, bei der die Bürger ihre Tugenden selbst erwerben und in konkreten Ordnungen reproduzieren. In ihren zentralen Passagen ist Aristoteles' politische Theorie auf die Vorstellung gegründet, die konkrete Ausgestaltung einer Verfassung hänge von den sozio-moralischen Dispositionen der Bürger ab, und umgekehrt sei bei der Ausgestaltung der Verfassung darauf zu achten, daß die für den Erhalt der Verfassung erforderlichen sozio-moralischen Ressourcen durch die politische Ordnung reproduziert würden. ${ }^{18}$ Auch Platon hält die sozio-moralischen Voraussetzungen der Bürger in seiner Zuordnung von Aufgaben und Ämtern sowie charakterlichen Dispositionen für wesentlich. Dabei läßt er aber innerhalb strikter Ordnungsvorstellungen und Erziehungskonzepte den Bürgern wenig Raum für eine eigenständige Entwicklung. Sein ideenzentriertes Konzept des Gemeinwohls koppelt dieses von einem demokratisch verstandenen Gemeinsinn ab; statt dessen avancieren die Philosophen zu privilegierten Interpreten des Gemeinwohls und die unter ihrem Einfluß stehenden Politiker zu dessen Dienern und Wächtern. In der Idee der Nächtlichen Versammlung hat Platon in den Nomoi sogar noch ein gesondertes Organ von Hütern des Gemeinwohls und der Verfassung entworfen. Seine in der Ideenlehre und auch in der Theologie verankerte substanzialistische Lösung kennt demnach eine Reihe von Gemeinwohlakteuren, die mit der Erkenntnis, der Realisierung und der Kontrolle des Gemeinwohls betraut sind.

Platon und Aristoteles stehen für unterschiedliche Muster einer verfassungstechnischen Sicherung des Gemeinwohls: Platon favorisiert eine hierarchische (etatistische) Lösung, in der das Gemeinwohl nur in einer Ordnung als ganzer realisiert werden kann, wobei sein Gehalt wesentlich von oben, durch die Philosophen und Regierenden, bestimmt und festgelegt wird. Bei Aristoteles dagegen sind, zumindest in der besten Ordnung, der Politie, Regierende und Regierte gleichermaßen an Festlegung, Realisierung und Kontrolle des Gemeinwohls beteiligt.

\section{Mittelalter, Kommunalismus, Reformation}

Wie die antike Begrifflichkeit im allgemeinen, so ist auch der Gemeinwohlbegriff lange Zeit durch den Filter der Thomasschen Kanonisierung des Aristoteles wahrgenommen worden. Für Thomas von Aquin war eine theologische Verankerung des bonum commune in dem auf eine transzendente Ordnung bezogenen summum bonum charakteristisch. Das Gemeinwohl wird dabei auf den verschiedenen Ebenen einer vielfach gestuften Ordnung identifiziert und definiert. Im Gegensatz zu dem Vermittlungsdenker Thomas betont Aurelius Augustinus, die andere, die Vorstellungswelt des Mittelalters prägende Gestalt, gerade den Gegensatz zwischen weltlichem und außerweltlichem Wohl. Dennoch treffen sich beide Denker darin, daß sie den Frieden auf der Ebene des Hauses, des politischen Gemeinwesens und zwischen politischen Gemeinschaften als eine Form des Gemeinwohls herausstellen.

18 Man kann die Freigabe des Soziomoralischen in allen modernen Verfassungen als Ausdruck einer Rechtsstaatlichkeit sehen, die sich im Unterschied zur aristotelischen Verfassungstheorie allein auf die Rechte der Bürger konzentriert und nur in Verbindung mit ihnen an wenigen Stellen auf konkrete Verpflichtungen zu sprechen kommt. 
Entgegen einer vor allem in ideen- und begriffsgeschichtlichen Arbeiten vorherrschenden Auffassung, betonen Historiker neuerdings, daß Thomas und Augustinus in nur geringem Ausmaß den Bezugspunkt bei der Neufassung und Säkularisierung des Gemeinwohls im späten Mittelalter gebildet haben. Nach Gerhard Oexle standen bereits im Mittelalter praktische Probleme, wie innere Sicherheit, Schutz vor Übergriffen und Friede, im Mittelpunkt des Bedeutungsfeldes von Gemeinwohl und gemeinem Nutzen. Den Zusammenhang von Gemeinwohl und Gemeinsinn veranschaulicht Oexle anhand mittelalterlicher Schwurgemeinschaften: In Kommunen, Gilden, Bruderschaften usw. wird ein Gruppengut via Vertrag bzw. Konsens als Gemeinwohl definiert, und durch einen Schwur verpflichten sich die Gruppenmitglieder auf seine Realisierung. Entscheidend ist dabei das Moment der Selbstverpflichtung. Begreift man die mittelalterliche Gesellschaft als einen in eine Vielzahl sozialer Gruppen fragmentierten Verband, so entsteht das Problem der Verknüpfung von gruppenbezogenen und allgemeinen Vorstellungen des Gemeinwohls. Diese Spannung wurde seinerzeit in Paradoxien, wie der von gewillkürtem Gruppenrecht und allgemeinem Recht, durchaus reflektiert und diskutiert. Das Gemeinwohl wurde zwar in einem theologischem Gesamtrahmen angesiedelt, politisch aber in den Ständen, sozialen Gruppen und Institutionen konkretisiert.

Mit Blick auf die Coniurationes kann auch eine generelle Differenz zum modernen Gemeinwohlverständnis aufgezeigt werden. Alle politischen Akteure agieren vor der Moderne nur auf eingeschränkte Weise öffentlich. Bei den höchstens auf lokale Teilöffentlichkeiten bezogenen und auch dann nur eingeschränkt öffentlichen Handlungen der Kommunen und Gilden ist der Schwur der wichtigste Akt in einer Teilöffentlichkeit und Bestandteil einer größeren Herrschaftsordnung. In diesem Kontext sind weitreichende Konsequenzen zu bedenken, die Peter von Moos jüngst wie folgt pointiert hat:

\begin{abstract}
„In politischer Hinsicht kann das Gebot der Skandalvermeidung wie eine ultima ratio wirken und die private Moral gegebenenfalls außer Kraft setzen. So kommentieren Rechtsgelehrte des 14. Jahrhunderts die vom Aquinaten getroffene Unterscheidung zwischen gerechter, zu Gehorsam verpflichtender und ungerechter, vom Gehorsam entbindender Obrigkeit: ,Wenn keine gerechte, sondern usurpierte oder ungerechte Herrschaft vorliegt, sind die Untertanen nicht gehalten zu gehorchen, es sei denn wegen der Vermeidung von Skandal und Gefahr' (nisi forte propter vitandum scandalum vel periculum). ,Die tyrannische Herrschaft ist ungerecht, weil sie nicht auf das Gemeinwohl (bonum commune), sondern auf das Privatwohl (bonum privatum) des Herrschers ausgerichtet ist; [ [... . Das im Prinzip zugestandene Widerstandsrecht gegen den Tyrannen wird mit dieser Einschränkung praktisch wieder aufgehoben, da eine Gehorsamsverweigerung, die keinen Skandal erregt, nichtöffentlich bleiben müßte, was nach aller politischen Erfahrung so gut wie unmöglich sein dürfte. Die vitatio scandalorum gehört somit wie das Gemeinwohl zu den Letztbegründungen gesellschaftlicher Ordnung, gegen die keine noch so edlen persönlichen Gewissensmotive geltend gemacht werden können." ${ }^{19}$
\end{abstract}

In der Moderne ist, so kann man im Gegenzug festhalten, das Gemeinwohl und seine Aushandlung an die Öffentlichkeit gebunden. Damit wächst der Skandalisierung ein völlig neuer Stellenwert zu und über diese rhetorisch-politische Strategie, die öffentliche Aufdeckung und Skandalisierung von verletztem Gemeinwohl, sind dessen inhaltli-

19 Vgl. dazu Moos 1998, S. 43f. 
che Momente oft am ehesten zu fassen. Das heißt im kontrastiven Vergleich: Während sich in der Moderne Akteure durch öffentliche Skandalisierung selbst zu Interpreten, Wächtern und Verwirklichern des Gemeinwohls erheben können, sind die Handlungsmöglichkeiten politischer Akteure durch das in die Gemeinwohlvorstellung eingeschriebene Gebot der Skandalvermeidung erheblich eingeschränkt. Zugespitzt läßt sich vielleicht sagen, daß die öffentliche Skandalisierung des Verhaltens und Handelns von Herrschenden partiell an die Stelle des Tyrannenmords getreten ist.

Für den Wandel in der Gemeinwohl-Semantik ist der sich seit dem 12. Jahrhundert entwickelnde Kommunalismus - im wesentlichen eine Bewegung kommunaler Selbstbestimmung auf städtischer, ländlicher und territorialer Ebene - von Bedeutung. In dieser Bewegung erfolgte, so Peter Blickle, eine Umarbeitung des feudalen HerrenNutzens in den kommunalen Gemeinen-Nutzen als eine Formel, die die Legitimation neuer Ordnungen und die Forderung des Friedens nach innen und außen zur Folge hatte. Dabei standen viel weniger aristotelische und thomistische Ideen im Vordergrund als eben konkrete Probleme, wie Schutz, Frieden und die Wahrung der Eigenständigkeit städtischer und ländlicher Kommunen. Erst mit dem ausgehenden 15., vor allem aber im 16. Jahrhundert im Gefolge der Reformation kam es schließlich zu einer „Verstaatlichung" des Gemeinwohls, in deren Gefolge sich die neuen Souveräne das Definitionsmonopol über das Gemeinwohl aneigneten.

War das Gemeinwohl im Mittelalter ein theologisch imprägnierter Begriff, dessen Säkularisierung und Entsubstanzialisierung schubweise erfolgte, so wird in der Reformation häufig eine theologische Wiederaufladung vorgenommen. Luther unterscheidet generell zwischen geistlichem und weltlichem Regiment. Gemeinwohl und gemeinen Nutzen, die für ihn letztlich ohne göttliches Wirken dauerhaft nicht gesichert werden können, denkt er, sobald es um die weltliche Ordnung geht, sehr konkret, nämlich im Rahmen seiner Ämter-, Stände- und Berufslehre, bei der Rollen, Institutionen und Personen deutlich unterschieden werden. ${ }^{20}$ Insofern verankert Luther das Gemeinwohl zwar substanzialistisch in der Theologie, alle weltlichen Ausfüllungen jedoch werden auf Strukturen und Institutionen bezogen, deren konkrete Kontexte wandelbar sind.

Man wird der Reformation freilich nur gerecht, wenn man in ihr einen Bruch mit der Vormoderne erkennt. In ihrem Selbstverständnis nämlich betreiben die Reformatoren erklärtermaßen eine doppelte öffentliche Skandalisierung des Gemeinwohlmißbrauches: durch die weltliche Herrschaft und durch die katholische Kirche. Insofern trägt die Reformation, trotz der Retheologisierung des Gemeinwohls, moderne Züge. Die Reformation verbleibt jedoch, was die Gemeinwohldefinition in der Öffentlichkeit betrifft, auf der Schwelle zur Moderne, denn die von ihr selbst genutzte Skandalisierung gemeinwohlschädlichen Verhaltens soll durch die praktisch realisierte Reformation nicht mehr nötig sein: Mit der Institutionalisierung der Visitationspraxis verliert die Öffentlichkeit als Medium der Unterbindung von gemeinwohlschädlichem Verhalten qua Skandalisierung wieder an Bedeutung.

Das Gemeinwohl wird in dieser Zeit durchaus mit verschiedenen Formen und Ebenen der Interessenaushandlung verknüpft, die allerdings ohne den Bezug auf ein höheres, allgemeines Interesse nicht auskommen. Nicht nur der Gedanke einer Schlichtungs

${ }^{20} \mathrm{Vgl}$. hierzu den Beitrag von Hans Grünberger. 
instanz spielt hierbei eine Rolle; vielmehr ist für den gesamten Gemeinwohldiskurs lange Zeit der Bezug auf einen nicht verfügbaren, höheren substanziellen Gesichtspunkt kennzeichnend. Wenn dieser wegfällt, d.h. wenn es keine theologischen, philosophischen oder monarchischen Gewißheiten mehr gibt, dann scheint Willkür unvermeidlich. Wenn daher die Entsubstanzialisierung des Gemeinwohls in der Reformation nur halbherzig betrieben wird, so sind dabei auch politische Ordnungsvorstellungen und Interessen von ausschlaggebender Bedeutung.

\section{Neubestimmungen in der Renaissance und der Frühen Neuzeit}

Eine erhebliche Vielfalt in der Begriffsbestimmung und operationalen Verwendung von Gemeinwohl läßt sich in der Renaissance und der Frühen Neuzeit beobachten, wobei diese Zeit auch darum von gesteigertem Interesse ist, weil in ihr die politische Sprache der Moderne vorgebildet wird. Für die Renaissance, zumal ihre italienische Variante, ist eine Verknüpfung von Gemeinwohl und Gemeinsinn vor allem im republikanischen Denken typisch. Die Grundüberlegung läßt sich wie folgt zusammenfassen: Eine Republik beruht auf bestimmten Tugenden der Bürger; die Prosperität des Gemeinwesens, die sich im Gefolge dieser Tugenden einstellt, kann aber dazu führen, daß das Engagement für das Gemeinwohl zugunsten privater Interessen allmählich abnimmt und so der Gemeinsinn erodiert. Die nach außen sichtbare Blüte einer Republik ist gegenüber dem sozio-moralischen Scheitelpunkt verspätet und stellt einen sub-optimalen Zustand dar, in dem sich der innere Verfall der sozio-moralischen Ressourcen, die die republikanischen Institutionen tragen, bereits eingestellt hat. Ein verstärkter Verfall der republikanischen Institutionen und der korrespondierenden Tugenden kann zwar aufgehalten und verzögert werden, aber er führt in der Regel zur Destruktion der politischen Form. Im Rahmen eines zyklischen Verfassungswechsels können dann in anderen Regimen wieder die Voraussetzungen für eine Republik und das Engagement der Bürger geschaffen werden. $^{21}$

Diese schon bei den römischen Historikern Sallust und Livius angelegte Linie wurde durch den italienischen Bürgerhumanismus neu gefaßt und insbesondere von Machiavelli so entwickelt, daß eine ganze Reihe moderner Denker, wie z.B. Montesquieu, an sie anknüpfen konnte. Die republikanische Strömung führt, wie John Pocock gezeigt hat, bis in den Diskurs der amerikanischen Verfassungsväter. ${ }^{22}$ Dabei kam es sukzessive zu einer Ablösung des Republikanismus vom Stadtstaat und seiner Anwendung auf institutionelle Flächenstaaten, für die vor allem die Founding Fathers der amerikanischen Republik und Tocqueville stehen. ${ }^{23}$ Für alle diese Autoren ist kennzeichnend, daß sie enge Verknüpfungen zwischen dem Gemeinwohl, den institutionellen Arrangements und den erforderlichen Handlungsqualitäten der politischen Akteure sowie ihren soziomoralischen Kompetenzen vornehmen. Es ist geradezu ein Kennzeichen des republikanischen Denkens, daß es aus dieser Verbindung die politische Dynamik, das Auf und

\footnotetext{
21 Vgl. Münkler 1991.

22 Vgl. Pocock 1975, Baron 1992.

${ }^{23} \mathrm{Zu}$ den Founding Fathers und zum Republikanismus von Jefferson vgl. den Beitrag von CordFriedrich Berghahn.
} 
$\mathrm{Ab}$ von politischen Gemeinwesen, zu erklären versucht hat. In die zyklische Geschichtsauffassung ist zugleich ein Funktionswandel der Gemeinwohlrhetorik eingeschrieben. Die öffentliche Debatte über das Gemeinwohl setzt in der Regel nämlich erst dann ein, wenn Verfall wahrgenommen wird, d.h. die Republik ihre äußerliche Blüte offensichtlich überschritten hat. Politischer Verfall wird dabei in der Klage des Tugendverlusts kommuniziert. ${ }^{24}$ In beiden Fällen kritisiert die republikanische Rhetorik zunächst die Praxis von Republiken und zielt auf deren Erneuerung u.a. durch die Einschränkung der Vermögensunterschiede in ihr. Wenn der Verfall zum Wechsel der Regierungsform geführt hat, wird die Kritik neu ausgerichtet und erlangt nun stellenweise revolutionäre Züge. Schließlich fungiert die Gemeinwohlrhetorik als Mobilisierungsideologie zu Schaffung und Aufbau einer Republik; vor allem in dieser Periode kann sie ihr herrschaftslimitierendes und die Bürger aktivierendes Potential entfalten. Der optimale Zustand einer Republik, durch nur mäßigen Reichtum und geringe Expansion nach außen gekennzeichnet, ist der, in dem nach diesem Modell am meisten Gemeinwohl und Gemeinsinn realisiert werden und gleichzeitig am wenigsten davon die Rede ist.

Neben dem Genre der Fürstenspiegel, in dem gemeinwohlförderliche Anforderungen an den Herrscher beschrieben werden, ${ }^{25}$ und den Utopien, die fiktiv verwirklichte Gemeinwohlordnungen ausmalen, trifft man in der politischen Theorie der Frühen Neuzeit auf systematische Konzepte, wie das von Hobbes, und auf Kritik an solchen Systembauten inklusive deren Theoremen über Gemeinwohl, wie sie etwa Montaigne verkörpert. Er hat in seinen Essais das Gemeinwohlkonzept als Tarnung von Ehrgeiz und Habgier gedeutet, gleichzeitig aber auch immer wieder mit affirmativen Bezug vom Gemeinwohl gesprochen. ${ }^{26}$ Diese Decouvrierung und gleichzeitige Inanspruchnahme des Gemeinwohls gehört in der Moderne, insbesondere bei den französischen Moralisten und den an sie anschließenden Autoren, zum Repertoire, mit dem öffentlich um das Gemeinwohl gestritten wird. Als Theoretiker ist freilich Thomas Hobbes viel wirksamer geworden. Er denkt die Politik von einem robusten Interessenkonzept mit dem Individuum als Nutzenmaximierer her und hat diesem Konzept zum Durchbruch verholfen. Dabei verzichtet er allerdings nicht auf den Gemeinwohlbegriff, legt aber die Verfügung darüber ausschließlich in die Hand des Souveräns. Das Gemeinwohl war damit rein säkular bestimmt und auch konzeptionell in hohem Maße politisch verfügbar geworden. Bislang taten die Theoretiker so, als gebe es „das Gemeinwohl“, und die politischen Praktiker instrumentalisierten es theoretisch. Nun stellt Hobbes, ähnlich im übrigen wie die Theoretiker der Staatsräson, ${ }^{27}$ das Gemeinwohl ganz bewußt in die Instrumentenkammer des Souveräns. Es gab allerdings nicht nur Ursurpationsversuche seitens

${ }^{24}$ Diese vor allem im Übergang vom 15. zum 16. Jahrhundert zu beobachtende Klage wiederholt sich in der zweiten Hälfte des 18. Jahrhunderts; vgl. Hirschman 1980, Hont/Ignatieff 1983 sowie Pocock 1985 , S. $37 \mathrm{ff}$.

$25 \mathrm{Vgl}$. Mühleisen u.a. 1997, insbes. S. 9-21.

${ }^{26}$ Zum verschiedenen Gebrauch der Gemeinwohlvokabel vgl. die Essais IX und X, in Montaigne 1992, Bd. III, 3. Buch.

${ }^{27}$ Zur Verbindung von Staatsräson und Gemeinwohl insbes. bei Herman Conring vgl. Stolleis 1988, S. $207 \mathrm{ff}$. 
des Souveräns, sondern auch durch die verschiedene Parteien des englischen Bürgerkrieges, die das Gemeinwohl, den Common Weal, zu ihren Zwecken zu nutzen trachteten. Neben im engeren Sinn religiösen Motiven spielte dabei auch das Interesse an freier politischer Verfügbarkeit der Gemeinwohlsemantik eine bedeutende Rolle. ${ }^{28}$

\section{Semantischer Coup des Liberalismus}

Bernard de Mandeville hat im frühen 18. Jahrhundert dem Gemeinwohldiskurs mit seiner Bienenfabel und ihrer These vom öffentlichen Nutzen privater Laster $^{29}$ eine neue Richtung gegeben. Er betreibt eine Substitution des Gemeinwohls durch die Eigeninteressen, eliminiert dabei aber das Gemeinwohl nicht komplett. Entscheidend ist, daß Mandeville auf seine Weise die Gesellschaft als einen besonderen Raum entdeckt hat. Er weist dem Staat und der Legislation eine distinkte Aufgabe zu, nämlich Rahmenbedingungen für das Gemeinwohl zu sichern. Die Pointe hierbei ist, daß es um eine entmoralisierte Politik geht, die sich nicht direkt in gesellschaftliche Belange einmischt und an ihren Effekten zu messen ist. Mandeville wird nicht selten in seinem Einfluß vor allem auch auf Adam Smith unterschätzt. Smith hat sich mehrfach mit ihm auseinandergesetzt und folgte immer mehr seinem Argument der ,private vices, public benefits“, das er jedoch nie in der paradoxen Form wie der Autor der Bienenfabel faßte. ${ }^{30}$

Smith ist besonders wirksam geworden, weil er mit anderen schottischen Autoren die Politische Ökonomie als neue wissenschaftliche Disziplin hervorbrachte, die mit der Moralphilosophie um den definitiven Einfluß auf die Verhaltenssteuerung der Menschen konkurriert: Die Moralphilosophie zielt dabei auf Motive und Intentionen, während die Politische Ökonomie bei Effekten und Ergebnissen ansetzt. Die Rezeption seiner Theorien, die sich als eine Abfolge der Aufnahme und Universalisierung seines Argumentes der unsichtbaren Hand fassen läßt, erfolgte vor allem im 19. Jahrhundert. Dieses Argument besagt, daß das vielfache Verfolgen der wirtschaftlichen Eigeninteressen in einer Marktwirtschaft am Ende allen nutzt und zu einer sinnvollen Ordnung führt. Smith hatte dieses Argument eher en passant vorgebracht, dennoch ist hier im Prinzip eine Umkehr der Relation von Privatinteressen und Gemeinwohl vollzogen: Man muß das Gemeinwohl gar nicht mehr intentional anstreben, sondern es setzt sich in der Marktordnung aufgrund bestimmter institutionalisierter Regeln durch (diese Regeln werden allerdings nicht näher beschrieben). An die Stelle der Intentionalität politischer wie gesellschaftlicher Akteure, um die sich der Republikanismus gesorgt hatte, trat die Funktionalität institutionalisierter Märkte, die mit geringerem Aufwand und größerer Erwartungssicherung zu demselben Ergebnis zu führen beansprucht. Entscheidend ist, daß das Wohl der Menschen hierbei auf materielle Interessen reduziert und nicht mehr innerhalb einer Vielzahl von Leidenschaften gefaßt wird. Mit Adam Smith rückt die Politische Ökonomie als wissenschaftliche Disziplin und der Experte als soziale Figur zur Bestimmung der richtigen, gemeinwohlförderlichen Ordnung in den Vordergrund.

\footnotetext{
${ }^{28} \mathrm{Vgl}$. zu den einzelnen Parteiungen und ihrer Inanspruchnahme des Gemeinwohls den Beitrag von Raimund Ottow.

${ }^{29}$ Vgl. Mandeville 1980, v.a. S. 84.

${ }_{30} \mathrm{Zu}$ Mandeville vgl. Euchner 1973, S. 74-131, sowie Hundert 1994.
} 
Man kann die bei Smith im Konzept der ,invisible hand" angelegte Umkehr des Zusammenhangs von Privat- und Gemeinwohl als „semantischen Coup des Liberalismus ${ }^{631}$ festhalten, und zwar insbesondere deshalb, weil diese Denkfigur nicht lange auf den Bereich der Wirtschaft beschränkt blieb. Immanuel Kant, nebenbei gesagt ein Bewunderer Mandevilles, hat sie paradigmatisch auf den Staat übertragen. Die Republik wird auf eine institutionalisierte Rechtsordnung gegründet, was selbst für ein „Volk von Teufeln (wenn sie nur Verstand haben) ${ }^{632}$ möglich sei. Damit treten die Intentionen der Akteure sowie deren sozio-moralische Dispositionen, auf denen die Institutionen aufruhen, in den Hintergrund. Diese Linie der Ersetzung des Gemeinwohls reicht über die Utilitaristen bis hin zu Mancur Olson und - in der deutschen Politikwissenschaft - zu Fritz Scharpf, der die Umstellung der Gemeinwohlkonzeption von einer Input- auf eine Output-Kontrolle am stärksten favorisiert hat. ${ }^{33}$ Hier soll nur ein Aspekt der semantischen Umstellung im 18. Jahrhundert angedeutet werden: Der neue Ausgangspunkt ist das Individuum; von seinen Rechten her wird das Gemeinwesen gedacht. In diesem Rahmen wird das Gemeinwohl als ein inhaltlich fixiertes Konzept substituiert, zumindest aber umgewertet. In Verbindung damit treten Freiheit, Sicherheit und Wohlstand in den Mittelpunkt der Gemeinwohlvorstellung, die zunehmend nicht mehr vom Souverän, vom Staat, sondern vom Individuum her gedacht wird.

\section{Verstaatlichung des Gemeinwohls in der Policey-Wissenschaft}

Kant und Smith standen in Opposition zu einer Richtung, die ebenfalls an Hobbes anknüpft, aber nicht so sehr an sein Interessenkonzept als vielmehr an die Bindung des Gemeinwohls an den Souverän. Insbesondere die Policey-Wissenschaft im 17. und 18. Jahrhundert übersetzte dieses Konzept in eine durch vielfältige Ordnungen von oben geregelte Staatlichkeit. ${ }^{34}$ Dabei dienen Gemeinwohl und Gemeinsinn wesentlich als politische Vokabeln zur Passivierung der Bürger. Gleichzeitig wird mit diesen Konzepten, wie Thomas Simon zeigt, eine Verdichtung von Herrschaft erzielt, deren Versprechen mehr Sicherheit und Wohlfahrt sind. Michel Foucault pointiert diese Entwicklung:

„Bei Pufendorf heißt es: ,Man hat ihnen [den Souveränen] die souveräne Autorität nur übertragen, damit sie sich ihrer bedienen, um den öffentlichen Nutzen herbeizuführen und zu wahren.' Ein Souverän darf nichts für vorteilhaft für sich selbst halten, wenn es dies nicht auch für den Staat ist. Worin besteht aber nun dieses Gemeinwohl oder auch dieses Heil aller, von dem die Rechtsgelehrten sprechen und das regelmäßig als der eigentliche Zweck der Souveränität geltend gemacht und aufgestellt wird? [...]. Das öffentliche Wohl ist [...] im Wesentlichen der Gehorsam vor dem Gesetz, vor dem Gesetz des Souveräns über diese Erde oder vor dem Gesetz des absoluten Souveräns, Gott. Doch, wie auch immer, bezeichnend für den Zweck der Souveränität, für dieses Gemeinwohl oder allgemeine Wohl, ist letzten Endes nichts anderes als absolute Unterwerfung. Der Zweck der Souveränität ist somit zirkulär: Er verweist auf die

$31 \mathrm{Vgl}$. Münkler/Fischer 1999, S. 247f.

${ }^{32}$ Kant 1995, S. 224.

33 Scharpf 1991.

34 Maier 1966, Stolleis 1988, S. $334 \mathrm{ff}$. 
tatsächliche Ausübung der Souveränität; das Wohl ist der Gehorsam vor dem Gesetz, demnach ist das Wohl, das die Souveränität sich vornimmt, daß die Leute ihr gehorchen. "35

Damit ist ein paradoxer Status des Gemeinwohlbegriffes von Unbestimmtheit und groBer Bestimmtheit beschrieben: Es handelt sich um einen im Prinzip entsubstanzialisierten Begriff, der nur auf den Gehorsam gegenüber dem Souverän abstellt, aber gleichzeitig ist dieser durch eine Vielzahl von Ordnungen und Verordnungen gebunden, in denen das Gemeinwohl konkretisiert ist.

Historisch liegt der genannten Uminterpretation des Gemeinwohls eine Intensivierung von Herrschaft und eine Steigerung von Integration zugrunde, die durch PoliceyOrdnungen geregelt wird. Auf ökonomischem Gebiet ist dabei die Herausbildung von Territorial-Ökonomien wesentlich. Die „Lands-Würthschaften“ stellen solche Einheiten dar, die mit merkantilistischen Politiken reguliert werden. Dabei erfolgt auf seiten der Politik eine Umstellung der Wissensformen: Statt der personal verkörperten Weisheit von Regenten und Beratern sind nun Experten und wissenschaftlich gestützte Argumente von Ökonomen und Juristen gefragt, wie dies Wolf-Hagen Krauth zeigt. Hinzu kommt, daß für die Durchsetzung der Policey-Ordnungen nicht einfach ein hobbesianisches Deutungsmonopol des Gemeinwohls seitens des Souveräns genügt, sondern eine Beamtenschaft, die es en detail interpretiert und durchsetzt.

Wie wichtig es bei der Analyse von Gemeinwohlvorstellungen ist, Semantiken mit Praktiken zu verbinden, wird bei einem Blick auf die Genese des neuzeitlichen Staates deutlich: Waren Gemeinwohl und Gemeinsinn in der kommunalen Bewegung Selbstbehauptungsvokabeln, die als Semantik reflexive Praktiken der Ausübung der Freiheit der Bürger begleiteten und sich historisch auf ziviles und militärisches Engagement bezogen, so wird das Gemeinwohl nun zu einer Passivierungs- und Disziplinierungsformel von Herrschaftspraxen, wie Matthias Bohlender betont. Aus einem Reflexionsbegriff von Bürgerschaft wird Gemeinwohl zu einer Verfügungsvokabel von Herrschaft. Dabei wurden im Ordnungsschema von „Policey“ gesellschaftliche, sittliche und politische Formen zusammengebracht, die dem Ziel der Mehrung des Gemeinwohls dienen sollen. Es versteht sich von selbst, daß dabei der Gemeinsinn der Bürger in den Hintergrund rückt. Das heißt freilich nicht, daß sich die kommunale Tradition gänzlich erschöpft hätte. Vielmehr wird sie länderspezifisch in Europa unterschiedlich stark kontinuiert, und immer wieder dient die Gemeinwohlsemantik auch als Medium bürgerschaftlicher Selbstermächtigung gegen obrigkeitliche Passivierung. Dennoch kommt mit der Ausbildung des institutionellen Flächenstaates die Verstaatlichung der Gemeinwohlkompetenz erst richtig zum Zuge. In diesem Kontext wird auf staatlicher Ebene die Grenze privat-öffentlich paternalistisch von „oben“ gezogen. Inhaltlich erfolgt dabei eine Verschiebung der Themen; wiewohl die Ordnung das Thema ist, geht es hinsichtlich der Individuen viel weniger um das gute Leben oder das wohlgeordnete Gemeinwesen, sondern in den Vordergrund rücken Sicherheit, Wohlstand und konkrete, staatlich gewährte Freiheitsspielräume.

\footnotetext{
${ }^{35}$ Foucault 2000, S. 53.
} 


\section{Kritik am liberalen Modell im 19. Jahrhundert}

Brachte das 19. Jahrhundert im Zeichen von industrieller Umwälzung und politischen Revolutionen den eigentlichen Siegeszug liberaler Ideen, so gibt es in ihm aber auch theoretische Reaktionen auf die individualistische Substitution der Gemeinwohl- und Gemeinsinnsemantik, die innerhalb einer Rezeption der Politischen Ökonomie und der liberalen politischen Theorie vorgenommen werden. Dies läßt sich exemplarisch an Hegel, Lorenz von Stein und Otto von Gierke zeigen, die alle mehr oder weniger zu einer organisch-kommunitären Linie politischen Denkens gehören. Diese Autoren gehen vom modernen Individuum und damit von der Annahme aus, das gesellschaftliche Leben sei wesentlich Kampf. Zugleich wird das Gemeinwohl bei ihnen weder als kleinster gemeinsamer Nenner noch als Nebenprodukt egoistischen Handelns gedacht, sondern als ein Ganzes, das mehr als die Summe der Teile ist. Interessant sind diese Entwürfe auch darum, weil sie unter modernen Bedingungen mehr oder weniger explizit an Gemeinwohlvorstellungen festhalten und zugleich auf eine limitierte Weise eine Art von Pluralismus denken. Das beginnt bei der Annahme einer Vielfalt von Gemeinwohlakteuren, die an der Bestimmung des Allgemeinwohls beteiligt sind. Das Gemeinwohl wird in einer subsidiär gegliederten Ordnung gefaßt, so daß es mehrere Gemeinwohle auf unterschiedlichen Ebenen gibt sowie ein Gemeinwohl, das sich auf die Ordnung als Ganze bezieht. Die problematische Seite dessen ist freilich die Verselbständigung des Staates zum Hüter und Garanten des Gemeinwohls schlechthin. Der Staat als Sphäre und Hüter des Allgemeinen wird von der Sphäre der Wirtschaft, der bürgerlichen Gesellschaft, deutlich unterschieden, aber nicht getrennt von ihr gedacht. Hegel akzentuiert in der Sphäre der bürgerlichen Gesellschaft die Korporationen als integrative und intermediäre Institutionen; Stein betont das aufgeklärte Sonderinteresse der herrschenden Klasse; und Gierke hebt hervor, auch im Privatrecht müsse die Orientierung auf das Gemeinwohl eingelassen sein, ebenso wie das Privatwohl im öffentlichen Recht systematisch zu berücksichtigen sei. ${ }^{36}$

Hegel, Stein und Gierke favorisieren ein Konzept gestuften Gemeinwohls verschiedener Gemeinschaften, deren Verbindung in einer Gesamtordnung nach dem Prinzip der Subsidiarität gedacht wird. ${ }^{37}$ Die entsprechenden Vorstellungen von Interessenvermittlung stellen nicht nur auf institutionelle Einbindungen ab, die dazu führen, daß auch private Interessen immer schon gesellschaftlich bestimmt, zumindest reflektiert werden, sondern sie zielen auf eine Vermittlung von einzelnen, besonderen und allgemeinen Interessen, die zu einem Bestandteil der gesellschaftlichen und politischen Integration wird. Weil diese Vermittlung in institutionelle Regelungen eingebunden ist, werden in ihr immer wieder auch die Voraussetzungen gemeinwohlorientierten Handelns thematisch. Das Konzept gestuften Gemeinwohls enthält eine treffende Kritik am liberalindividualistischen Konzept, das Ordnungsbildung nur als Nebeneffekt betrachtet und insofern selbst die Bedingungen individueller Interessenverfolgung nur partiell berücksichtigt.

\footnotetext{
36 Vgl. Hegel 1981, Stein 1959, Bd. 1-3, Gierke 1889, S. 6. Zu Stein vgl. Böckenförde 1991.
}

${ }^{37}$ Dazu Riklin/Batliner 1994. 
Denkt Hegel den Staat in einer gegliederten Ordnung nach einem Interessenläuterungsmodell, so bedarf es doch zugleich einer besonderen sozialen Gruppe als Hüter, Interpreten und Realisatoren des Gemeinwohls. Diese Rolle übernimmt die Beamtenschaft, der „allgemeine Stand“, wie Hegel sie nennt. Die Rolle ist allerdings keineswegs so exklusiv gedacht, wie eine staatszentrierte Hegel-Interpretation gemeint hat, denn dieser Stand ist nicht nur in eine institutionelle Ordnung eingebettet, sondern bedarf auch noch der Korporationen als Hort des Allgemeinen in der bürgerlichen Gesellschaft, also auf vorstaatlicher Ebene. Als Vermittlungsdenker sieht Hegel „subsidiäre“ Formen von Gemeinwohlsicherung in der bürgerlichen Gesellschaft vor, die allerdings unter Rechtsaufsicht des Staates zu stellen sind. ${ }^{38}$

Viel stärker als Hegel betont Stein im Hinblick auf die akute soziale Frage den Einfluß der Gesellschaft, wodurch die Aufgabe des Staates, Garant des Allgemeinen zu sein, und die freie Entwicklung aller zu ermöglichen, umfassender, aber auch problematischer wird. Stein akzentuiert, daß die Legitimität moderner Staaten an den tagtäglichen Leistungen der Verwaltung gemessen werde. Insofern hebt auch er auf die Reproduktion nichtinstitutioneller Voraussetzungen staatlicher Institutionen ab. Insgesamt gesehen reformulieren und modernisieren Hegel und Stein zu einem erheblichen Teil die polizeiwissenschaftlichen Argumentationen, jedoch nicht mit der Absicht einer Passivisierung der Bürger, sondern gerade in aktivierender Absicht. ${ }^{39}$

Demgegenüber hebt Otto von Gierke die Notwendigkeit einer Vielzahl von Verbänden hervor. Er strebt eine ,Zurückverlegung des Staates in das Volk ${ }^{\text {“40 }}$ an und faßt unter dem Titel der Genossenschaft kommunitäres Engagement über Hegel und Stein hinausgehend als gleichwertiges Gegenstück zur Herrschaft. Dies ist auch die Grundlage seiner angelsächsischen pluralismustheoretischen Rezeption. ${ }^{41}$ Wenn Gierke wie Stein am Staat als realer und nicht-fiktiver Persönlichkeit festhält, so nicht zuletzt mit Blick auf die größere Gewißheit und reelle Allgemeinheit, die dem Staat im Vergleich mit den Verbänden zukommt. Statt den Staat in eine Fiktion aufzulösen, wie es mancher liberale Autor tut, hat eine sozialwissenschaftliche Deutung des Staates, getreu dem Thomas-Theorem, dem zufolge alle Fiktionen, die geglaubt werden, wirksam sind, den Staat als besondere Größe an Glauben und Tun politisch-gesellschaftlicher Akteure zurückzubinden. Das heißt aber zugleich, den Staat als Hüter und Garanten des Allgemeinwohls auf eine politische Kultur und Praxis zu gründen; seine Wesensbestimmung und sein Wirken sind dann an kontingente Umstände geknüpft, die zu kontrollieren und zu reproduzieren ihm nur begrenzt möglich ist.

Die Idee des Gemeinwohls und auch ihre Verknüpfung mit dem Gemeinsinn bleibt, wie anhand unterschiedlicher theoretischer Strömungen des 19. Jahrhundert skizziert,

\footnotetext{
38 Hegel, Rechtsphilosophie $\S 235$.

39 Dies kann man nicht nur an Hegels Abschnitten zur Polizei und Rechtspflege in der Rechtsphilosophie ablesen, sondern auch in der Anlage und Regelungsdichte, die sein Staatsrecht vorsieht. Stein hat sich in seiner Verwaltungslehre (1865-68) explizit mit der Polizeiwissenschaft auseinandergesetzt.

40 Gierke 1889 , S. 10.

$41 \mathrm{Vgl}$. Runciman 1997.
} 
eine aktuelle Begrifflichkeit der modernen politisch-sozialen Sprache. ${ }^{42}$ Festzuhalten ist in diesem Zusammenhang, daß die Theoretiker sich und ihre Disziplinen (Philosophie, Gesellschafts- und Verwaltungslehre, Rechtswissenschaft) als besondere Hüter, Wächter und Interpreten des Gemeinwohls selbst privilegieren, wobei es immer wieder zu einem Wechsel in der Position der in besonderer Weise mit der Pflege des Gemeinwohls betrauten Disziplinen kommt. Hinsichtlich der historischen Transformation der Gemeinwohlsemantik im 19. Jahrhundert sind zwei Punkte interessant. Zum einen die überwölbende Tendenz der Nationalisierung des Gemeinwohls, die sowohl von „oben“, durch den Staat, als auch von ,unten“, durch die Bürger und die nationale Bewegung, erfolgt. Die politische Idee der Nation beruht auf einer generellen Aktivierung ihrer Mitglieder, auf einem spezifischen Bürgersinn, der durch Gleichheits- und Solidaritätsversprechen gewonnen wird. Der Staat und die nationalen Eliten müssen dafür im Tausch nicht nur Schutz, Rechtssicherheit und ein bestimmtes Ausmaß an Gleichheit und Solidarität sichern, sondern sie können im nationalen Interesse (ein Substitutsbegriff für nationales Wohl) auch Solidaritätszumutungen und Opferforderungen an die Bürger richten. Es hängt von der spezifischen Ausprägung der Nationen, von ihrer inneren Verfassung wie ihrer geschichtlichen Entstehung ab, inwieweit es dabei auf nationaler und/oder darunter liegenden Ebenen (Regionen, Kommunen) zu Verknüpfungen zwischen Gemeinwohl und Gemeinsinn kommt.

Zum zweiten kennzeichnet das 19. Jahrhundert ein andauerndes und selten näher betrachtetes Fortwirken der republikanischen Tradition, die mit neuen Inanspruchnahmen der Gemeinwohlsemantik, insbesondere durch bürgerliche Schichten, einhergeht, wofür der Aufschwung des Stiftungswesens und des Mäzenatentums im Kaiserreich, den $\mathrm{Ma}$ nuel Frey darstellt, neben anderen Geselligkeitsformen und Assoziationen ein guter Indikator sind. Geht man diskursanalytisch vor, dann stellt sich das 19. Jahrhundert mit seinem Vereinswesen, seiner Blüte der Freimauerer-Bewegung, seiner bürgerlichzivilen Geselligkeit, als eine Zeit dar, in der - statt seines oft diagnostizierten Endes ein eigener Tugenddiskurs gepflegt wird. So gesehen, bleibt diese Ära noch im Schatten frühneuzeitlicher Begriffe, wie Stefan-Ludwig Hoffmann zeigt, und sie kontinuiert den republikanischen Denkstrang, in dem Gemeinwohl und Gemeinsinn zusammen gedacht wurden.

Fragt man nach diesem selektivem Gang durch die Geschichte des politischen Denkens im Hinblick auf einige Eckpunkte der Semantik von Gemeinwohl und Gemeinsinn, was ihren Anspruch, ein Bestandteil moderner Leitbegrifflichkeit zu sein, rechtfertigen kann, so ist es vor allem ihre schwache inhaltliche Bestimmtheit und die daraus resultierende vielfältige operationale Verwendbarkeit. Beides läßt sie immer wieder für verschiedene soziale und politische Akteure attraktiv erscheinen. Man kann politische Aufgaben und Projekte positiv mit dem Gemeinwohl verknüpfen oder ex negativo in Abgrenzung zu gemeinwohlschädigendem Verhalten thematisieren. Der skizzierte theoretische Ansatz eröffnet eine erfolgversprechende Verbindung von Gemeinwohl und

${ }^{42}$ Um so bemerkenswerter ist, daß ein einschlägiger Artikel in den Geschichtlichen Grundbegriffen, dem von Brunner, Conze und Koselleck herausgegebenen „Historischen Lexikon zur politischsozialen Sprache in Deutschland" fehlt. 
Gemeinsinn in dem Sinne, daß man damit die überkommenen Konnotationen von Gemeinwohl als einem substanzialistischen Begriff aufsprengen und mit der Gemeinwohlsemantik transportierte Problemstellungen jenseits des alten begrifflichen Horizontes erörtern kann. Die Verbindung von politischen Ideen, politischer Rhetorik und praktisch-institutionellen Definitions- sowie Realisierungsprozessen erlaubt, die Transformation des Gemeinwohls und seine Wandlungen im Zusammenhang mit dem Gemeinsinn zu markieren und in ihrer Bedeutung für die Selbstthematisierung von Gesellschaften herauszustellen. Der Kampf um Begriffe und Ideen ist, wie der Wandel der Semantik zeigt, immer auch ein Kampf um die politische Hegemonie. Die auf eine Aktivierung des Gemeinsinnes der Bürger zielende Nutzung des Gemeinwohlkonzeptes ist in der Regel herrschaftslimitierend, während die Bestimmung des Gemeinwohls von „oben“, die auf eine Passivierung der Bürger zielt, herrschaftslegitimierend fungiert. In historisch-politischen Diskursen prallen diese Varianten nicht nur oft aufeinander, sondern es entstehen auch Mischformen, die oft nicht leicht zu durchschauen sind. Thematisch werden in all diesen Fällen die Handlungsorientierungen von Bürgern und politischen Gemeinwesen, die Ermöglichung kooperativen Handelns und die Reproduktion der dafür nötigen institutionellen und sozio-moralische Ressourcen.

\section{Literatur}

Alemann, U. v./Heinze, R. G./Wehrhöfer, U. (Hg., 1999), Bürgergesellschaft und Gemeinwohl. Analyse, Diskussion, Praxis, Opladen.

Baron, H. (1992), Bürgersinn und Humanismus im Florenz der Renaissance, Berlin.

Bell, D. (Hg., 1993), Communitarianism and Its Critics, Oxford.

Böckenförde, E.-W. (1991): Lorenz von Stein als Theoretiker der Bewegung von Staat und Gesellschaft zum Sozialstaat, in: Ders., Recht, Staat, Freiheit. Studien zur Rechtsphilosophie, Staatstheorie und Verfassungsgeschichte, Frankfurt/M., S. 170-208.

Dahl, R. A. (1989), Democracy and Its Critics, New Haven/London.

Deth, J. W. van/Maraffi, M./Newton, K./Whiteley, P. F. (1999), Social Capital and European Democracy, London.

Euchner, W. (1973), Egoismus und Gemeinwohl. Studien zur Geschichte der bürgerlichen Philosophie, Frankfurt/M.

Fischer, K. (2000), Gemeinwohlrhetorik und Solidaritätsverbrauch. Bedingungen und Paradoxien des Wohlfahrtsstaates, in: Ethik im Sozialstaat, hg. v. M. Prisching, Wien, S. 131- 154.

Foucault, M. (2000), Die „Gouvernementalität“, in: Gouvernementalität der Gegenwart. Studien zur Ökonomisierung des Sozialen, hg. v. U. Brökling u.a., Frankfurt/M., S. 41-67.

Gebhardt, J./Münkler, H. (Hg. 1993), Bürgerschaft und Herrschaft. Zum Verhältnis von Macht und Demokratie im antiken und neuzeitlichen politischen Denken, Baden-Baden.

Gierke, O. v. (1889), Die soziale Aufgabe des Privatrechtes, Berlin.

Gunn, J. A. W. (1969), Politics and the Public Interest in the Seventeenth Century, London/Toronto.

Hegel, G. W. F. (1981), Grundlinien der Philosophie des Rechtes oder Naturrecht und Staatswissenschaft im Grundrisse, Berlin.

Hibst, P. (1991), Utilitas Publica - Gemeiner Nutz - Gemeinwohl. Untersuchungen zur Idee eines politischen Leitbegriffes von der Antike bis zum späten Mittelalter, Frankfurt/M. u.a. 
Hirschman, A. O. (1980), Leidenschaften und Interessen. Politische Begründungen des Kapitalismus vor seinem Sieg, dt. von S. Offe, Frankfurt/M.

Hirschman, A. O. (1984), Engagement und Entscheidung. Über das Schwanken der Bürger zwischen Privatwohl und Gemeinwohl, dt. von S. Offe, Frankfurt/M.

Hirschman, A. O. (1989), Moral und Sozialwissenschaften. Über die Langlebigkeit ihres Spannungsverhältnisses; in: Ders., Entwicklung, Markt und Moral. Abweichende Betrachtungen, Frankfurt/M.

Hirschman, A. O. (1997), Grenzübertritte, in: Ders., Tischgemeinschaft. Zwischen öffentlicher und privater Sphäre. Jan Patocka-Gedächtnisvorlesung des IWM 1996, Wien, S. 34-76.

Hofmann, H. (2000), Einführung in die Rechts- und Staatsphilosophie, Darmstadt.

Honneth, A. (Hg., 1993), Kommunitarismus: Eine Debatte über die moralischen Grundlagen moderner Gesellschaften, Frankfurt/M./New York.

Hont, I./Ignatieff, M. (Hg., 1983), Wealth and Virtue. The Shaping of Political Economy in the Scottish Enlightment, Cambridge.

Hundert, E. G. (1994), The Enlightenment's Fable. Bernard Mandeville and the Discovery of Society, Cambridge/New York.

Kant, I. (1995), Zum ewigen Frieden. Ein philosophischer Entwurf, in: Immanuel Kant Werkausgabe, hg. v. W. Weischedel, Frankfurt/M., Bd. IX, S. 193-251.

Kaufmann, M. (1999), Gemeinwohl und Recht, in: Das Gemeinwohl zwischen Universalismus und Partikularismus. Zur Theorie des Gemeinwohls und der Gemeinwohlwirkung von Ehescheidung, politischer Sezession und Kirchentrennung, hg. von P. Koslowski, Stuttgart/Bad Cannstatt, S. 102-123.

Kempshall, M. S. (1999), The common good in late medieval thought, Oxford.

Klages, H. (1999), Individualisierung als Triebkraft bürgerschaftlichen Engagements. Empirische Fakten und Folgerungen, in: Perspektiven gesellschaftlichen Zusammenhalts, hg. v. E. Kistler u.a., Berlin, S. 101-111.

Koselleck, R. (1972), Einleitung, in: Geschichtliche Grundbegriffe. Historisches Lexikon zur politisch-sozialen Sprache in Deutschland, hg. v. O. Brunner, W. Conze, R. Koselleck, Stuttgart, S. XIII-XXVII.

Koselleck, R. $\left(1995^{3}\right)$, Vergangene Zukunft. Zur Semantik geschichtlicher Zeiten, Frankfurt/M.

Koslowski, P. (Hg., 1999), Das Gemeinwohl zwischen Universalismus und Partikularismus. Zur Theorie des Gemeinwohls und der Gemeinwohlwirkung von Ehescheidung, politischer Sezession und Kirchentrennung, Stuttgart/Bad Cannstatt.

Ladd, E. C. (1999), The Ladd Report, New York.

Maier, H. (1966), Die ältere deutsche Staats- und Verwaltungslehre, Neuwied a. Rhein/Berlin.

Mandeville, B. de (1980), Die Bienenfabel oder Private Laster, öffentliche Vorteile, Frankfurt/M.

Miller, P. N. (1994), Defining the Common Good: Empire, Religion and Philosophy in Eighteenthcentury Britain, Cambridge/New York/Oakleigh.

Montaigne, M. de (1992), Essais, Zürich, Bd. 1-3.

Moos, P. v. (1998), Das Öffentliche und das Private im Mittelalter. Für einen kontrollierten Anachronismus, in: Das Öffentliche und das Private in der Vormoderne, hg. von G. Melville und P. v. Moos, Köln/Weimar/Wien, S. 3-83.

Mühleisen, H.-O./Stammen, Th./Philipp, M. (Hg., 1997), Fürstenspiegel der Frühen Neuzeit, Frankfurt/M.

Münkler, H. (1991), Die ldee der Tugend. Ein politischer Leitbegriff im vorrevolutionären Europa, in: Archiv für Kulturgeschichte, 73. Bd., S. 379-403. 
Münkler, H. (1997), Der kompetente Bürger, in: Politische Beteiligung und Bürgerengagement in Deutschland, hg. v. A. Klein und R. Schmalz-Bruns, Baden-Baden, S. 153-172.

Münkler, H. (2000), Ehre, Amt und Engagement. Wie die knappe Ressource Bürgersinn gesichert werden kann, in: Forschungsjournal Neue soziale Bewegungen, Heft 2, S. 22-32.

Münkler, H. (Hg., 1996), Bürgerreligion und Bürgertugend. Debatten über die vorpolitischen Grundlagen politischer Ordnung, Baden-Baden.

Münkler, H./Fischer, K. (1999), Gemeinwohl und Gemeinsinn. Thematisierung und Verbrauch soziomoralischer Ressourcen in der modernen Gesellschaft; in: Berlin-Brandenburgische Akademie der Wissenschaften, Berichte und Abhandlungen Bd. 7, Berlin, S. 237-265.

Münkler, H./Fischer, K./Bluhm, H. (2000), Das Ende einer semantischen Karriere? Zur Gegenbegrifflichkeit von Gemeinwohl und politischer Korruption, in: Berlin-Brandenburgische Akademie der Wissenschaften, Berichte und Abhandlungen Bd. 8, Berlin, S. 425-440.

Münkler, H./Krause, S. (2001), Der aktive Bürger; in: Politik im 21. Jahrhundert, hg. v. C. Leggewie und R. Münch, Frankfurt/M. (i.E.).

Offe, C. (1999), „Sozialkapital“". Begriffliche Probleme und Wirkungsweise, in: Perspektiven gesellschaftlichen Zusammenhalts, hg. v. E. Kistler, u.a., Berlin, S. 113-120.

Pocock, J. G. A. (1975), The Machiavellian Moment. Florentine Political Thought and the Atlantic Republican Tradition, Princeton (N.J.).

Pocock, J. G. A. (1985), Virtue, Commerce and History. Essays on Political Thought and History, Chiefly in the Eighteenth Century, Cambridge.

Putnam, R. D. (1995), Bowling Alone: America's declining social capital, in: Journal of Democracy, Jan. 1995, Vol. 6, S. 65-78.

Putnam, R. D. (2000), Bowling Alone. The Collapse and Revival of American Community, New York.

Putnam, R. D. (Hg., 2001), Gesellschaft und Gemeinsinn. Sozialkapital im internationalen Vergleich, Gütersloh.

Riklin, A./Batliner, G. (Hg., 1994), Subsidiarität, Baden-Baden.

Runciman, D. (1997), Pluralism and the Personality of the State, Cambridge.

Scharpf, F. W. (1991), Die Handlungsfähigkeit des Staates am Ende des 20. Jahrhunderts, in: Politische Vierteljahresschrift, 32. Jg., Heft 4, S. 621-634.

Scharpf, F. W. (1999), Regieren in Europa. Effektiv und demokratisch?, Frankfurt/M.

Skocpol, Th. (1997), The Tocqueville Problem. Civic Engagement in American Democracy, in: Social Science History 21: 4 (1997), S. 455-479.

Stein, L. v. (1865-68), Verwaltungslehre, 7 Theile, Stuttgart.

Stein, L. v. (1959), Geschichte der sozialen Bewegung in Frankreich, Darmstadt, Bd. 1-3.

Stolleis, M. (1988), Geschichte des öffentlichen Rechts in Deutschland. Bd. 1: 1600-1800, München.

Walzer, M. (1992), Zivile Gesellschaft und amerikanische Demokratie, Berlin. 